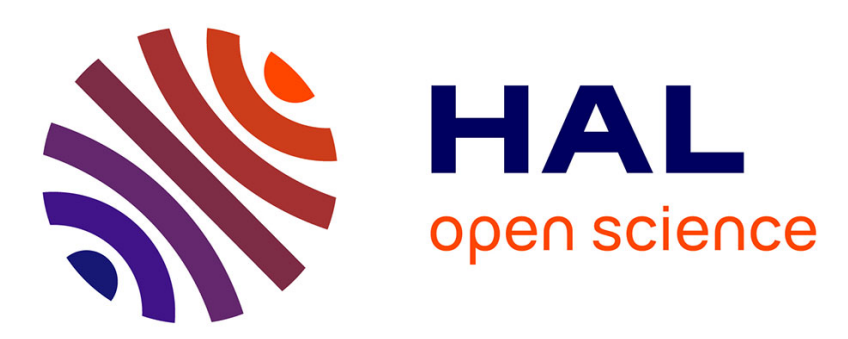

\title{
Reliability importance analysis of Markovian systems at steady state using perturbation analysis
}

\author{
Phuc Do Van, Anne Barros, Christophe Bérenguer
}

\section{To cite this version:}

Phuc Do Van, Anne Barros, Christophe Bérenguer. Reliability importance analysis of Markovian systems at steady state using perturbation analysis. Reliability Engineering and System Safety, 2008, 93, pp.1605- 1615. 10.1016/j.ress.2008.02.020 . hal-00705179

\section{HAL Id: hal-00705179 \\ https://hal.science/hal-00705179}

Submitted on 7 Jun 2012

HAL is a multi-disciplinary open access archive for the deposit and dissemination of scientific research documents, whether they are published or not. The documents may come from teaching and research institutions in France or abroad, or from public or private research centers.
L'archive ouverte pluridisciplinaire HAL, est destinée au dépôt et à la diffusion de documents scientifiques de niveau recherche, publiés ou non, émanant des établissements d'enseignement et de recherche français ou étrangers, des laboratoires publics ou privés. 


\title{
Reliability importance analysis of Markovian systems at steady state using perturbation analysis
}

\author{
Phuc Do Van, Anne Barros, Christophe Bérenguer \\ Université de technologie de Troyes \\ Institut Charles Delaunay - FRE CNRS 2848 \\ Systems Modeling and Dependability Group \\ 12, rue Marie Curie - BP 2060 -10010 Troyes cedex - France \\ Tel : +33 325718027 - Fax: +33 325715649 \\ Corresponding author: Anne Barros \\ E-mail : anne.barros@utt.fr
}

\begin{abstract}
ABSTRACT: Sensitivity analysis has been primarily defined for static systems, i.e. systems described by combinatorial reliability models (fault or event trees). Several structural and probabilistic measures have been proposed to assess the components importance. For dynamic systems including inter-component and functional dependencies (cold spare, shared load, shared ressources, ....), and described by Markov models or, more generally, by discrete events dynamic systems models, the problem of sensitivity analysis remains widely open. In this paper, the perturbation method is used to estimate an importance factor, called multi-directional sensitivity measure, in the framework of Markovian systems. Some numerical examples are introduced to show why this method offers a promising tool for steady state sensitivity analysis of Markov processes in reliability studies.
\end{abstract}

Keywords: perturbation analysis; sensitivity analysis; importance measure; Markov process; dynamic system 


\section{Introduction}

The sensitivity analysis of the results of a system reliability study (i.e. reliability importance analysis) helps to identify which components contribute the most to system (un)performance (reliability, maintainability, safety, or any other performance measure). Hence, the reliability sensitivity analysis provides fruitful insight into the system behavior, helps to find design weaknesses or operation bottlenecks and to suggest optimal modifications for system upgrade (improved design, better maintenance, ...). To take full advantage of reliability studies, it is thus of great importance to have at one's disposal efficient sensitivity analysis methods which can be implemented on industrial systems, without oversimplifying assumptions.

Sensitivity analysis has been well defined and investigated for static systems, i.e. systems with independent components described by combinatorial reliability models (fault or event trees). Several structural and probabilistic measures have been proposed to assess components' importance [17, 19]. Most of these measures are linked to each other and the Birnbaum importance, defined as the partial derivative of the system availability with respect to the availability of its components is one of the most widely used. A well established methodology exists to compute the sensitivity measures, the most efficient being based on binary decision diagrams (BDD), [11].

For dynamic systems including inter-component and functional dependencies (cold spare, shared load, shared resources, ....), and described by Markov models or, more generally, by discrete events dynamic systems models, the problem of sensitivity analysis remains widely open. The primary objective of this paper is thus to propose, in the context of Markov process modelling and stationary performance measure, an importance measure based on partial derivative with respect to a parameter, rather than to the availability of a component. This measure (called multi-directional sensitivity measure) can be an efficient tool to investigate not only the importance of a given component, but also the importance of a class of components, the importance of a system state, and, more generally, the effect of the simultaneous change of several design parameters that are related to the components or the system state. Then, a calculation method is proposed by the use of Perturbation Analysis and one of its variants, Perturbation Realization $[6,7]$. The aim is to show that the multi-directional sensitivity measure can be estimated with realistic feedback data set: it is supposed that the infinitesimal generator of the Markov process is unknown, the operational condition of the system has no need to be modified to evaluate the impact 
of the change of one parameter, the size of the system can be quite considerable. Hence the present work relies on the joint use of the multi-directional sensitivity measure as an importance factor and Perturbation Realization as an estimation method for this factor.

The paper is organized as follows: section 2 presents the main issue of the paper, that is the problem of importance reliability analysis in case of inter-dependent components, the proposition of a new importance measure in the context of Markov process at steady state, and the study of its properties. Section 3 is devoted to the evaluation of this importance measure by the use of Perturbation Realization approach. At last, two specific numerical examples are provided in section 4 to show why this method offers a promising tool for steady state sensitivity analysis of Markov processes in reliability studies. Finally, section 5 presents the conclusions drawn from this work.

\section{Notation list}

A asymptotic availability of the system

$D \quad$ realization matrix

$\hat{D} \quad$ estimated realization matrix

$d_{i j} \quad$ realization factor

$\eta_{t}\left(M, X_{t}^{l}\right)$ performance function of process $X_{t}^{l}$ at time $t$

$\bar{\eta}_{t}(M)$ performance measure at time $t$

$\bar{\eta}(M) \quad$ limit of $\bar{\eta}_{t}(M)$

$\mathbb{I}_{(A)} \quad$ indicator function (equals 1 when event $\mathrm{A}$ is true)

$I_{Q} \quad$ multi-directional importance measure

$I_{Q}^{C} \quad$ multi-directional importance measure at the component level

$I_{Q}^{S} \quad$ multi-directional importance measure at the system level

$\lambda, \mu \quad$ failure and repair rate of one unit

$M \quad$ transition rate matrix

$n \quad$ number of system parameters

$\pi \quad$ stationary probabilities vector

$Q \quad$ directional perturbation matrix

$S \quad$ discrete state space

$\theta \quad$ system parameters set

$W^{*} \quad$ minimum level required of system performance 


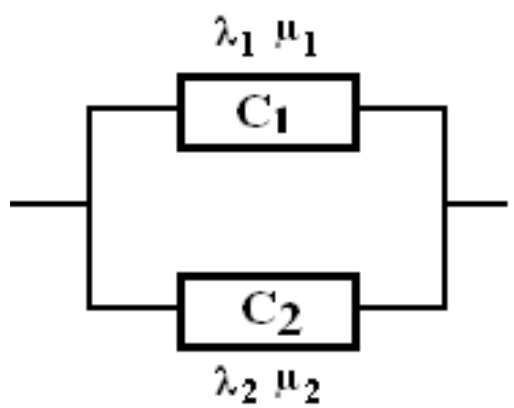

Figure 1: A parallel structure of 2 independent units

\section{Sensitivity analysis of Markovian systems: prob- lem statement}

\subsection{Classical importance measures}

Markov models are frequently used in reliability analysis to assess different measurements of interest, e.g. system reliability, availability, maintainability. Within this Markov modelling framework, the traditional reliability importance factors (e.g. Birnbaum importance or critical importance factor) used to analyze the system performance sensitivity with respect to the failure availability of its components can be computationally expensive to evaluate. Moreover, in the context of dynamic Markovian systems with inter-component and functional dependencies (cold spare, shared load, shared resources, ...), even the meaning and the definition of these traditional importance factors become questionable.

Take for example a system consisting of two independent units $C_{1}$ and $C_{2}$ in a parallel structure with constant failure rates $\lambda_{1}, \lambda_{2}$ and constant repair rates $\mu_{1}, \mu_{2}$ (see Figure 1). The transition matrix of this system is given by:

$$
M=\left(\begin{array}{cccc}
-\lambda_{1}-\lambda_{2} & \lambda_{1} & \lambda_{2} & 0 \\
\mu_{1} & -\mu_{1}-\lambda_{2} & 0 & \lambda_{2} \\
\mu_{2} & 0 & -\mu_{2}-\lambda_{1} & \lambda_{1} \\
0 & \mu_{2} & \mu_{1} & -\mu_{1}-\mu_{2}
\end{array}\right)
$$

The system availability at steady state equals:

$$
A=1-\left(1-a_{1}\right)\left(1-a_{2}\right)
$$


where $a_{i}$ is the steady state availability of component $\mathrm{i}$, and it is written as:

$$
\begin{aligned}
a_{i} & =\lim _{t \rightarrow+\infty}\left(\frac{\mu_{i}}{\lambda_{i}+\mu_{i}}+\frac{\lambda_{i}}{\lambda_{i}+\mu_{i}} e^{-\left(\lambda_{i}+\mu_{i}\right) t}\right) \\
& =\frac{\mu_{i}}{\lambda_{i}+\mu_{i}}
\end{aligned}
$$

In this case, the traditional importance measures as Birnbaum's importance measures [23], defined as the derivative of the system availability with respect to the availability of a given component in the context of independent components system, are directly calculable:

$$
\begin{aligned}
& I^{B}\left(C_{1}\right)=\frac{\partial A}{\partial a_{1}}=1-a_{2} \\
& I^{B}\left(C_{2}\right)=\frac{\partial A}{\partial a_{2}}=1-a_{1}
\end{aligned}
$$

Next assume that dependencies exist between the components. It is the case for example when in a two-unit system $C_{2}$ is in cold redundancy with $C_{1}$, or when the failure rate of $C_{2}$ (resp. $C_{1}$ ) is switched to a higher value when $C_{1}$ (resp. $C_{2}$ ) fails, because of a shared load (Figures 2 and 3). Then the definition of an importance factor as the partial derivative of the system availability with respect to a component availability may become questionable. Indeed, the availability of a component does not depend only on

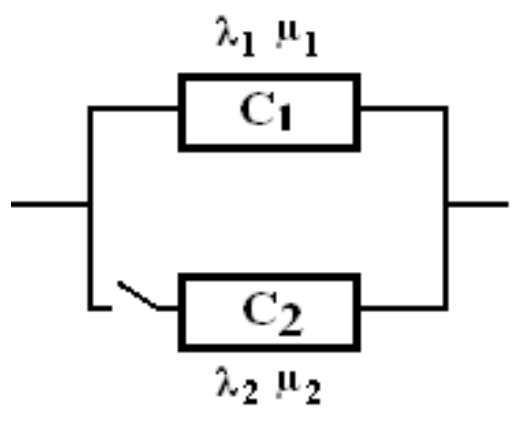

Figure 2: Cold redundancy structure

its characteristics but also on other system parameters, and its availability in the system can be different from its availability out of the system [20]. In this context, the partial derivative with respect to a set of parameters, rather than to the availability of a component, appears to be more relevant. The aim of this work is to propose an importance measure (called multi-directional sensitivity measure) that offers an interesting (and generalizing) alternative, especially for dynamic Markovian systems with inter-component 


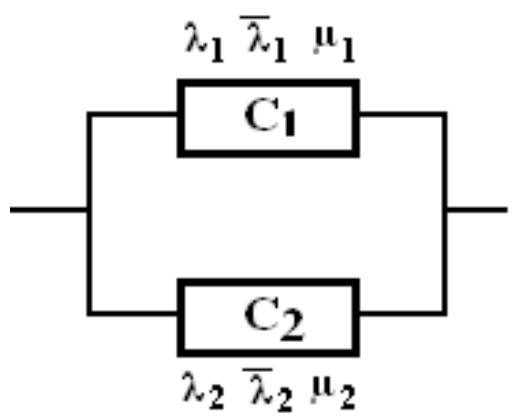

Figure 3: Shared load structure

dependencies.

\subsection{Proposition for the multi-directional sensitivity mea-} sure

The multi-directional sensitivity measure is proposed in this paper to study the sensitivity of the performance of Markovian systems at steady state. The first step is to define the performance criterion. In the previous example, it is equal to the availability of the system. In the following, a more general type of performance criterion is considered that corresponds to a linear function of the steady state probabilities.

\subsubsection{Performance function}

Consider an irreducible homogeneous Markov process with a finite state space. This process is, as a consequence, ergodic and a single stationary distribution exists [24]. This Markov process denoted $X_{t}^{l}$ is characterized by:

- a finite state space $S=\{1,2, \ldots, m\}$,

- an initial state $l \in S$,

- a set of parameters denoted $\theta$ which represents the transition rates, and determines the transition rates matrix denoted $M$ ( $M$ does not depend on time since $X_{t}^{l}$ is an homogeneous Markov process),

- $\pi=\left(\pi_{1}, \pi_{2}, \ldots, \pi_{m}\right)$, a row vector that represents the steady-state probabilities of the Markov process. 
The considered performance functions associate a state or a group of states with a real number. They are denoted $\eta_{t}\left(\theta, X_{t}^{l}\right)=\eta_{t}\left(M, X_{t}^{l},\right)$ and can be written as:

$$
\eta_{t}\left(M, X_{t}^{l}\right)=\sum_{k=1}^{m} \alpha_{k} \mathbb{I}_{\left(X_{t}^{l}=k\right)}
$$

where $\alpha_{k}$ is a real number. The stationary performance measure of the Markov process is often defined as the limit of the expected performance function:

$$
\bar{\eta}(M)=\lim _{t \rightarrow+\infty} E_{\pi}\left\{\eta_{t}\left(M, X_{t}^{l}\right)\right\}
$$

Taking the chosen performance function:

$$
\bar{\eta}(M)=\pi f
$$

where $f=\left[\alpha_{1}, \ldots, \alpha_{k}\right]^{T}$. Hence only the performance measures $\bar{\eta}(M)$ that are a linear function of the steady state probabilities are considered (for example mean times as MTTF and MTTR are excluded) .

\subsubsection{Multi-directional sensitivity measure (MDSM)}

\section{Definition}

Consider now a perturbation on one or more parameters of a Markov process that is equivalent to a perturbation in the transition rates matrix $M$. Hence, the transition matrix $M$ changes to:

$$
M_{\delta}=M+\delta Q,
$$

where $\delta$ is a real number and $Q=\left[q_{i j}\right]$ is a matrix representing the direction of perturbation: $q_{i j}$ equals 0 indicates that the matrix entry $M_{i, j}$ is not perturbed and a number $\beta$ different from 0 indicates that the matrix entry $M_{i, j}$ is perturbed by an amount $\beta \delta$. The only condition on the structure of $Q$ is that the matrix $M_{\delta}$ is also a transition matrix i.e. the sum of each row equals 0. According to Chapman-Kolmogorov equations, at steady state:

$$
\begin{aligned}
& \pi M=0 \\
& \pi_{\delta} M_{\delta}=0
\end{aligned}
$$




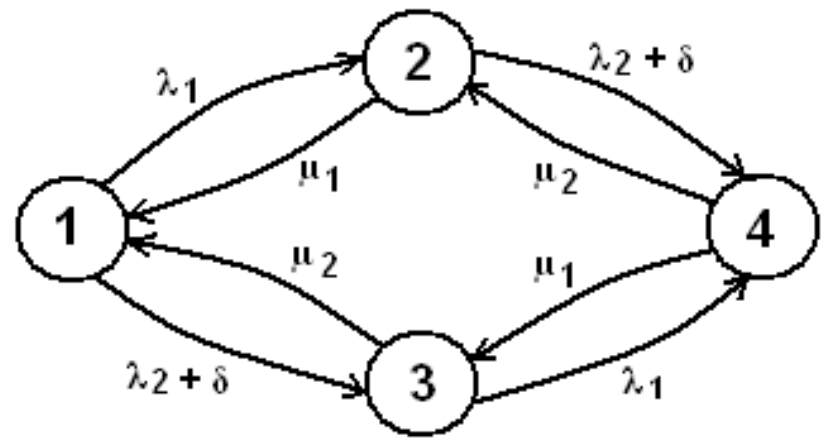

Figure 4: Perturbation on $\lambda_{2}$

$$
\begin{array}{ll}
\text { State } 1: C_{1} C_{2} & \text { State } 2:{ }_{C_{1}} \bar{C}_{2} \\
\text { State } 3: \overline{C_{1}} C_{2} & \text { State } 4: \overline{C_{1}} \\
C_{2}
\end{array}
$$

The stationary performance measure of the perturbed Markov process (that is the Markov process with transition matrix $\left.M_{\delta}\right)$ is denoted $\bar{\eta}\left(M_{\delta}\right)=\pi_{\delta} f$. Consequently, the derivative of $\bar{\eta}(M)$ in the direction of $Q$ can be defined as:

$$
\frac{d \bar{\eta}(M)}{d Q}=\lim _{\delta \rightarrow 0} \frac{\bar{\eta}\left(M_{\delta}\right)-\bar{\eta}(M)}{\delta} .
$$

The aim is to propose the use of the system performance derivative with respect to $Q$ as an importance factor. It is called multi-directional sensitivity measure (MDSM) in the following and denoted $I_{Q}$ :

$$
I_{Q}=\frac{d \bar{\eta}(M)}{d Q}
$$

\section{Two types of MDSM: $I_{Q}^{C}$ and $I_{Q}^{S}$}

Consider the previous example on Figure 1. The state diagram of this system is sketched in Figures 4 and 5 for two different types of perturbations. Figure 4 sketches the Markov graph with a perturbation on one specific parameter, namely $\lambda_{2}$, which corresponds to the directional perturbation matrix $Q_{1}$ :

$$
Q_{1}=\left(\begin{array}{cccc}
-1 & 0 & 1 & 0 \\
0 & -1 & 0 & 1 \\
0 & 0 & 0 & 0 \\
0 & 0 & 0 & 0
\end{array}\right)
$$

Figure 5 presents the Markov graph modified by a perturbation on the exit transition 


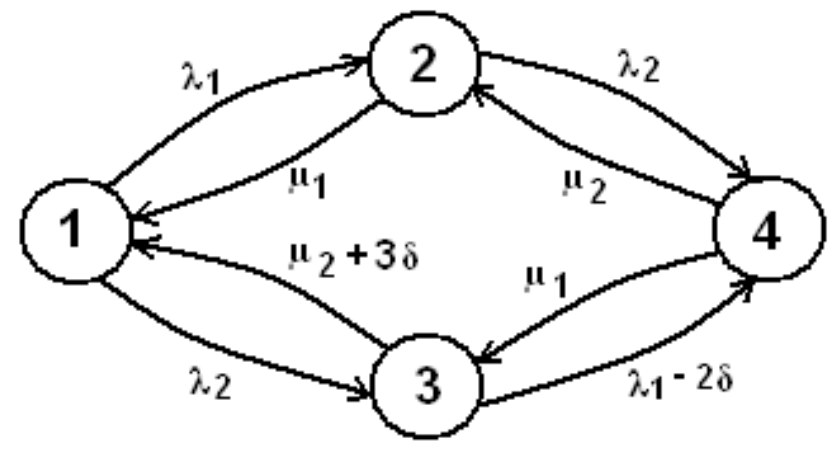

Figure 5: Perturbation on the exit transition rates of state 3

rates of one specific state, namely state number 3 . This perturbation corresponds to the directional perturbation matrix $Q_{2}$ :

$$
Q_{2}=\left(\begin{array}{cccc}
0 & 0 & 0 & 0 \\
0 & 0 & 0 & 0 \\
3 & 0 & -1 & -2 \\
0 & 0 & 0 & 0
\end{array}\right)
$$

The sensitivity measure $I_{Q_{1}}$ quantifies the importance of the parameter $\lambda_{2}$, which is the failure rate of a specific component. It gives the impact of a component failure on the system performance and:

$$
I_{Q_{1}}=\frac{d \bar{\eta}(M)}{d Q_{1}}=\frac{\partial \bar{\eta}(M)}{\partial \lambda_{2}}
$$

More generally, the partial derivative of $\bar{\eta}(M)$ with respect to parameter $\lambda_{i}$ corresponds to a particular $I_{Q\left(\lambda_{i}\right)}$ calculated with the perturbation matrix $Q\left(\lambda_{i}\right)=\left[q_{k j}\right]$ where $q_{k j}$ equals 1 if the parameter $\lambda_{i}$ appears in the matrix entry $M_{k j}$, and $q_{k j}$ equals 0 otherwise. In this way, the MDSM is close to the usual importance measures such as Birnbaum's, which are built with partial derivatives.

On the contrary, the sensitivity measure $I_{Q_{2}}$ quantifies the importance of a transition rate from one state to another one. It is a kind of conditional importance factor that quantifies the sensitivity to component $C_{2}$ failure rate, knowing that the system is in state 3. It is more connected to an importance measure at the system states level than at the components level. In this case, there is no direct connection with traditional importance measures.

In the following, if necessary, $I_{Q}^{C}$ refers to MDSM at the components level, and $I_{Q}^{S}$ to 
MDSM at the system states level. In particular, $I_{Q\left(\lambda_{i}\right)}^{C}$ refers to the partial derivative of the performance function with respect to parameter $\lambda_{i}$.

\subsection{Properties of the MDSM}

\subsubsection{MDSM and classical importance factor}

Consider the example in Figure 1 and take as performance function $\bar{\eta}(M)=A$, the system availability. $I_{Q_{1}}=I_{Q\left(\lambda_{2}\right)}^{C}$ corresponds to $\partial A / \partial \lambda_{2}$, i.e. the partial derivative of the system availability with respect to $\lambda_{2}$. In this case, the link with the Birnbaum's importance measure is directly established using the chain rule:

$$
\begin{gathered}
I^{B}\left(C_{2}\right)=\frac{\partial A}{\partial a_{2}} \\
=\frac{\partial A}{\partial \lambda_{2}} / \frac{\partial a_{2}}{\partial \lambda_{2}} \\
I^{B}\left(C_{2}\right)=I_{Q_{1}} / \frac{\partial a_{2}}{\partial \lambda_{2}}=I_{Q\left(\lambda_{2}\right)}^{C} / \frac{\partial a_{2}}{\partial \lambda_{2}} .
\end{gathered}
$$

In the case of a system with inter-dependent components as in Figure 2, the link between MDSM and Birnbaum's importance measure cannot be directly established since the latter is no longer defined. However, a MDSM of $I_{Q}^{C}$ type gives the same kind of information as a Birnbaum factor, since it can provide partial derivatives with respect to any system parameters.

\subsubsection{Relation with the transition matrix}

The particular structure of the Chapman-Kolmogorov equations and the linearity of the performance measure lead to the following expression of the measure derivatives [4]:

$$
\frac{d \bar{\eta}(M)}{d Q}=-\pi Q M^{*} f
$$

where $M^{*}$ is the inverse group of $M$ defined as:

$$
M^{*}=(M-e \pi)^{-1}-e \pi,
$$

where $e$ is a column vector of size $(n, 1)$ with $e_{i, 1}=1$ for any $i$. 


\subsubsection{Joint sensitivity}

Because of the linear structure of Equation 6, if a perturbation matrix $Q$ is a linear function of elementary perturbation matrixes $Q_{i}$, it is possible to evaluate the multidirectional importance measure related to $Q$ on the basis of the elementary perturbation measures related to the $Q_{i}$. For instance in the case of the system in Figure 1, for the joint sensitivity of the group of parameters $\left(\lambda_{1}, \lambda_{2}\right)$, it is possible to define a joint perturbation matrix:

$$
Q\left(\lambda_{1}, \lambda_{2}\right)=Q\left(\lambda_{1}\right)+Q\left(\lambda_{2}\right)
$$

where the matrix entry $q_{i, j}\left(\lambda_{k}\right)$ equals 1 if the parameter $\lambda_{k}$ is in the matrix entry $M_{i, j}$ and $q_{i, j}\left(\lambda_{k}\right)$ equals 0 otherwise. Then:

$$
\begin{aligned}
I_{Q\left(\lambda_{1}, \lambda_{2}\right)}^{C} & =\frac{\partial \bar{\eta}(M)}{\left.\partial Q\left(\lambda_{i}, \lambda_{j}\right)\right)} \\
& =-\pi Q\left(\lambda_{i}, \lambda_{j}\right) M^{*} f \\
& =-\pi\left(Q\left(\lambda_{i}\right)+Q\left(\lambda_{j}\right)\right) M^{*} f \\
& =-\pi Q\left(\lambda_{i}\right) M^{*} f+Q\left(\lambda_{j}\right) M^{*} f \\
& =I_{Q\left(\lambda_{i}\right)}^{C}+I_{Q\left(\lambda_{j}\right)}^{C}
\end{aligned}
$$

In this way, the joint sensitivity to a group of parameters can be the sum of the sensitivity measures to each parameter of the group. More generally, if a joint perturbation matrix with a different derivative direction for each parameter of a group of $n$ parameters is defined as:

$$
Q\left(\alpha_{1} \lambda_{1}, \alpha_{2} \lambda_{2}, \ldots, \alpha_{n} \lambda_{n}\right)=\sum_{i=1}^{n} \alpha_{i} Q\left(\lambda_{i}\right)
$$

then:

$$
\begin{aligned}
I_{Q\left(\alpha_{1} \lambda_{1}, \alpha_{2} \lambda_{2}, \ldots ., \alpha_{n} \lambda_{n}\right)} & =-\pi\left(\alpha_{1} Q\left(\lambda_{1}\right)+\alpha_{2} Q\left(\lambda_{2}\right)+\ldots \alpha_{n} Q\left(\lambda_{n}\right)\right) M^{*} f \\
& =\sum_{i=1}^{n} \alpha_{i} I_{Q\left(\lambda_{i}\right)}
\end{aligned}
$$

This property of the joint sensitivity cannot directly be connected to the additivity property of the differential importance measure presented by Borgonovo \& al. in $[1,2]$ : it concerns perturbation directions but not the components. In some particular cases, i.e. when the sensitivity measure related to one component can be defined by only one 
perturbation direction, the joint sensitivity to a group of components can be expressed as the sum of the sensitivity to the components. But it is not true as a rule and especially for system under consideration in this paper, i.e. with stochastic dependences.

As a conclusion, if the MDSM related to elementary perturbation directions are calculated, the joint MDSM related to any linear combination of these directions requires no additional calculations.

\section{Multi-directional sensitivity measure calcula- tion}

Many solutions have been proposed in literature to evaluate sensitivity measures corresponding to partial derivatives. Exact solutions rely on Frank's approach in [12]: the classical set of differential equations is extended to a bigger set of equations including the sensitivity factor equations. However, this approach is computationally burdensome and almost unusable or highly inefficient on realistic-size systems because the state space dimension is too great. To cope with this problem, some approximate solutions have been proposed but they are often only applicable to a limited class of systems (e.g. acyclic Markov models with no repair), [20].

Many simulation methods have been also proposed to estimate derivative measure. The simplest methods rely on the use of finite differences (FD) [8], and simultaneous perturbation (SP) [25]. These methods imply the change of the parameters for each simulation, which can be numerically burdensome and unfeasible for many real world systems, especially for those that need to be reliable. Methods based on common random numbers [8] offer a solution to this problem but if the value of the perturbation is too small, the resulting difference estimator could be severely affected by interference. The derivative estimation based on likelihood ratios (LR) has been proposed in $[16,14]$, and weak derivatives (WD) was introduced by Pflug [21, 22]. These methods provide an unbiased estimator, which leads to faster convergence rates when implemented in a simulation optimization algorithm, e.g., stochastic approximation [14].

In the framework of reliability studies, the perturbation analysis (PA) [3] and its variants, infinitesimal perturbation analysis (IPA), smoothed perturbation analysis (SPA)[13], structural IPA [9], Perturbation Realization [4] seem to be much promising. These meth- 
ods are based on the use of the stochastic gradient that can be estimated from one single sample path (i.e. without any change of the model parameters) [15]. Concerning Markov process modelling and stationary performance measure, Perturbation Realization is particularly well adapted $[6,7]$. It allows for:

- the evaluation of multi-directional sensitivity measures at the component level $\left(I_{Q}^{C}\right)$,

- the evaluation of multi-directional sensitivity measures at the system level $\left(I_{Q}^{S}\right)$,

- the evaluation of these sensitivity measures with operating feedback data, without any change of parameters, and without any knowledge of the infinitesimal generator of the Markov process.

\subsection{Introduction}

In the following, the main idea and the main steps of Perturbation Realization are presented. If:

- $X_{t}^{i}$, is a Markov process with transition matrix $M$ and initial state i,

- $X_{t}^{j}$, is a Markov process with transition matrix $M$ and initial state $\mathrm{j}$,

- $Y_{t}^{i, j}=\left(X_{t}^{i}, X_{t}^{j}\right)$,

- $K=\{(k, k), k \in S\}$,

and:

$$
T^{(i, j)}=\inf \left(t, t>0, Y_{t}^{i, j} \in K\right),
$$

then the realization factor $d_{i j}$ related to states $i$ and $j$ equals:

$$
d_{i j}=E\left\{\int_{0}^{T^{(i, j)}}\left[\eta_{t}\left(M, X_{t}^{j}\right)-\eta_{t}\left(M, X_{t}^{i}\right)\right] d t\right\}
$$

Consider a matrix $D$, called the realization matrix, so that $D(i, j)=d_{i j}$. Using a Lyapunov equation verified by $M$ and $D$, Cao showed in [4] that:

$$
I_{Q}=\frac{d \bar{\eta}(M)}{d Q}=\pi Q D^{T} \pi^{T}
$$

The derivative $d \bar{\eta}(M) / d Q$ is estimated on the basis of this equality. The advantages are that the inversion of matrix $(M-e \pi)$ in Equation 6 is avoided and that the matrix $D$ estimation relies on a single sample path. Hence, the realization factor defined for the 
steady state performance of a Markov process allows for, a direct estimation without matrix inversion, the estimation of the MDSM with a single sample path (from this point of view, this estimation method is part and parcel of Perturbation Analysis), and a very simple calculation of the MDSM for any perturbation matrix $Q$ (once the matrix $D$ is estimated).

Paragraph 3.2 is devoted to the interpretation of matrix $M$ and paragraph 3.3 to its estimation.

\subsection{Perturbation Realization factor}

In order to give an interpretation of the realization factor $d_{i j}$, consider the quantity $d_{i j}(\delta, \infty)$ so that:

$$
d_{i j}(\delta, \infty)=E\left\{\int_{t_{k}}^{\infty}\left[\eta_{t}\left(M_{\delta}, X_{t-t_{k}}^{\prime j},\right)-\eta_{t}\left(M, X_{t-t_{k}}^{i}\right)\right] d t\right\}
$$

with:

$t_{k}=$ the time upon which a perturbation occurs,

$X_{t-t_{k}}^{\prime j}$, a Markov process with transition matrix $M_{\delta}$, initial state $\mathrm{j}$ and initial time $t_{k}$,

$X_{t-t_{k}}^{i}$, a Markov process with transition matrix $M$, initial state i and initial time $t_{k}$,

$X_{t}^{l}$, a Markov process with transition matrix $M$, initial state 1 and initial time 0 .

Figure 6 gives an illustration for $X_{t-t_{k}}^{\prime j}, X_{t-t_{k}}^{i}, t_{k}$. First, there is a Markov process $X_{t}^{l}$, with initial state $l$ and transition matrix $M$. If, when the system is in state $k$, the transition rates $\lambda_{k j}$ is perturbed by an amount $+\delta$, it is possible that at the next transition time denoted $t_{k}$, the resulting perturbed Markov process $X_{t-t_{k}}^{\prime j}$ goes from state $k$ to state $j$ (perturbed sample path) instead of going from state $k$ to state $i$ as $X_{t}^{l}$ would do. In this case, the perturbation is "realized". If this is the only realized perturbation, then the impact of the perturbation on the performance measure is quantified by $d_{i j}(\delta, \infty)$. Then, since the Markov process is homogeneous:

$$
d_{i j}(\delta, \infty)=E\left\{\int_{t_{k}}^{\infty}\left[\eta_{t}\left(M_{\delta}, X_{t-t_{k}}^{\prime j}\right)-\eta_{t}\left(M, X_{t-t_{k}}^{i}\right)\right] d t\right\}
$$




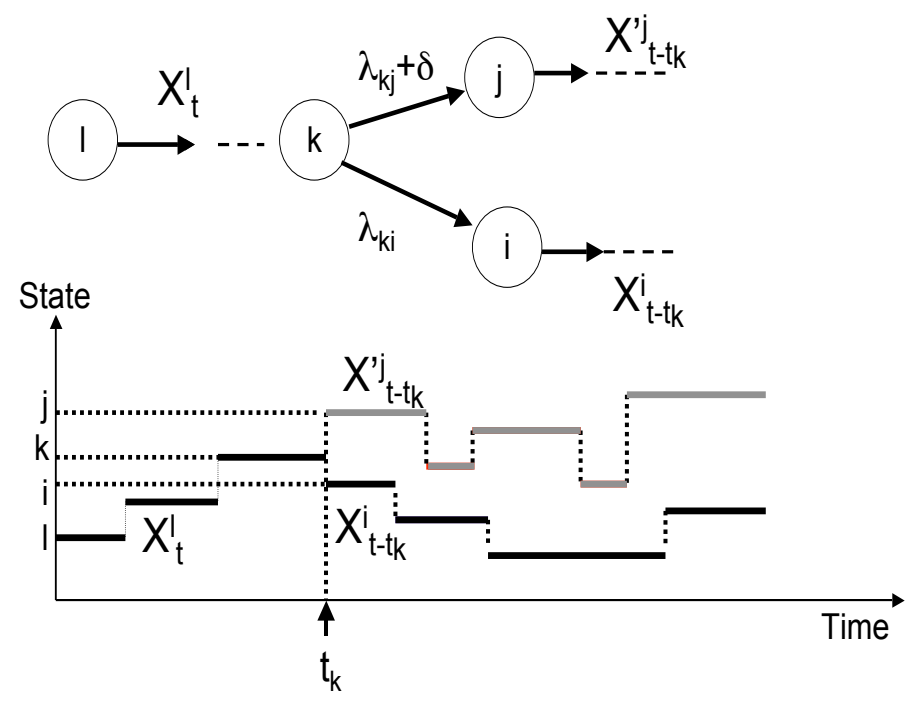

Figure 6: Perturbation Realization at time $t_{k}$

At the limit when $\delta$ approaches zero, (if the expectation and the limit can be permuted) :

$$
d_{i j}(\infty)=\lim _{\delta \rightarrow 0} d_{i j}(\delta)=E\left\{\int_{t_{k}}^{\infty}\left[\eta_{t}\left(M, X_{t-t_{k}}^{j}\right)-\eta_{t}\left(M, X_{t-t_{k}}^{i}\right)\right] d t\right\}
$$

where $X_{t-t_{k}}^{j}$ is a Markov process with transition Matrix $M$, initial state $j$ and initial time $t_{k}$. Finally, since the Markov processes $X_{t-t_{k}}^{j}$ and $X_{t-t_{k}}^{i}$ are ergodic and have the same transition matrix, there is a finite time $t_{e n d}$ upon which they merge in the same state $m$. Figure 7 sketches an illustration for $t_{e n d}, X_{t-t_{k}}^{j}$ and $X_{t-t_{k}}^{i}$. At time $t_{e n d}$, the perturbation vanishes since there are two Markov processes with the same transition rate and the same initial state. Then:

$$
E\left\{\int_{t_{\text {end }}}^{+\infty}\left[\eta_{t}\left(M, X_{t-t_{k}}^{j}\right)-\eta_{t}\left(M, X_{t-t_{k}}^{i}\right)\right] d t\right\}=0,
$$

and

$$
d_{i j}(\infty)=E\left\{\int_{t_{k}}^{t_{\text {end }}}\left[\eta_{t}\left(M, X_{t-t_{k}}^{j}\right)-\eta_{t}\left(M, X_{t-t_{k}}^{i}\right)\right] d t\right\}
$$

Since the Markov process is homogeneous:

$$
d_{i j}(\infty)=d_{i j}=E\left\{\int_{0}^{T^{(i, j)}}\left[\eta_{t}\left(M, X_{t}^{j}\right)-\eta_{t}\left(M, X_{t}^{i}\right)\right] d t\right\}
$$




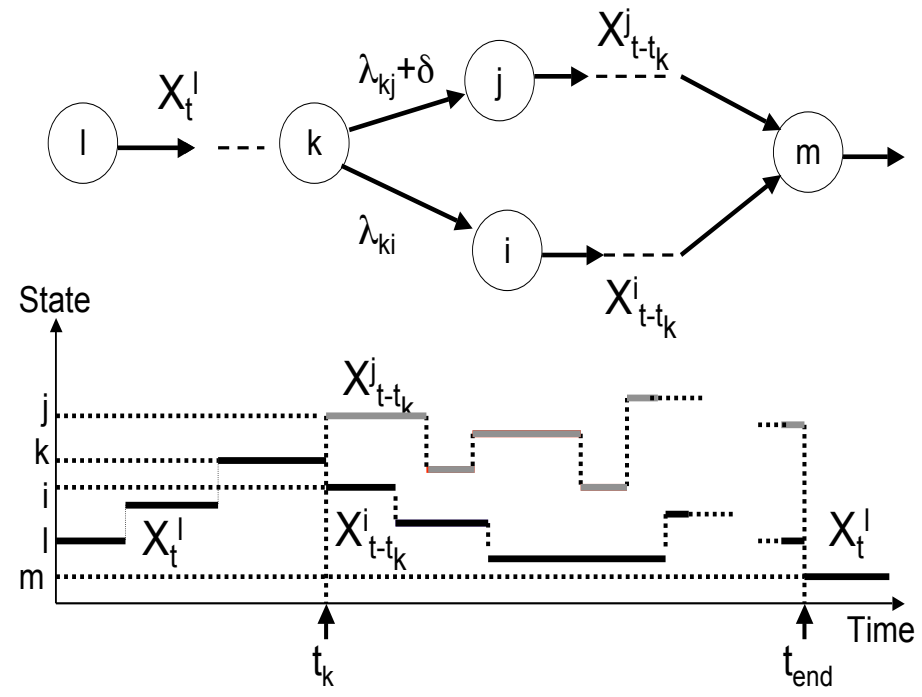

Figure 7: Illustration of the realization factor $d_{i j}$

As a result, $d_{i j}$ quantifies the impact of a unique perturbation on a sample path, when the process goes once to state $j$ (perturbed sample path), instead of going to state $i$ (nominal sample path). The main idea of the Perturbation Realization approach is to show that the evaluation of the impact of any number of perturbations over an infinite horizon (that is the calculation of the derivatives), can be made on the basis of the impact of only one perturbation (that is on the basis of the factor realizations $d_{i j, 1 \leq i \leq m, 1 \leq j \leq m}$ ).

\subsection{Derivatives estimate}

\section{Perturbation Realization factor estimate}

Cao showed in [4] that the Perturbation Realization factors can be estimated from a single sample path. Actually, if $\Gamma^{j}(i)$ is the first time upon which a Markov process with initial state $j$ arrives in state $i$, that is:

$$
\Gamma^{j}(i)=\inf \left\{t, t>0 / X_{t}^{j}=i\right\}
$$

then, if $E_{\pi}(f)<\infty$ and if $\lim _{t \rightarrow+\infty} E\left[\left|f\left(X_{t}^{i}\right)\right|\right]=E_{\pi}(|f|)$ :

$$
d_{i j}=E\left\{\int_{0}^{\Gamma^{j}(i)}\left[\eta_{t}\left(M, X_{t}^{j}\right) d t\right]\right\}-\bar{\eta}(M) E\left\{\Gamma^{j}(i)\right\}
$$


The first passage time $\Gamma^{j}(i)$ and the stationary probability vector $\pi$ (and consequently $\bar{\eta}(M))$ can be estimated from $X^{j}=\left(X_{t}^{j}, t \geq 0\right)$ as follows. A Markov process $X_{t}^{l}$ (initial state $l$ and transition matrix $M)$ is observed with the two time sequences $\left\{j_{s}\right\}$ and $\left\{i_{s}\right\}$ so that:

- $i_{0}=0$,

- $j_{s}=$ is the time upon which $X_{t}^{l}$ is in state $j$ for the first time after time $i_{s-1}$,

- $i_{s}=$ is the time upon which $X_{t}^{l}$ is in state $i$ for the first time after time $j_{s}$,

- $L_{s}^{j}(i)=i_{s}-j_{s}$ for $s \geq 1$ (independent and identically distributed),

- $R_{s}=\int_{k=j_{s}}^{i_{s}-1} \eta_{t}\left(X_{k}^{l}\right) d t$ (independent and identically distributed).

Then $\Gamma^{j}(i), \int_{0}^{\Gamma^{j}(i)}\left[\eta_{t}\left(X_{t}^{j}\right) d t\right]$, and $\bar{\eta}(M)$ are estimated by $\hat{\Gamma}^{j}(i), \hat{I}$, and $\hat{\bar{\eta}}(M)$ respectively

$$
\begin{aligned}
& \hat{\Gamma}^{j}(i)=\frac{1}{n} \sum_{s=1}^{n} L_{s}^{j}(i), \\
& \hat{I}=\frac{1}{n} \sum_{s=1}^{n} R_{s}, \\
& \hat{\bar{\eta}}(M)=\hat{\pi} f \text { with } \hat{\pi}_{i}=\frac{1}{N} \sum_{k=1}^{N} \mathbb{I}_{\left(X_{t_{k}}^{l}=i\right)},
\end{aligned}
$$

where $n$ is the number of observed sequences from $j$ to $i, N$ is the total number of observed sequences and $t_{k}$ is the $k$-th transition time. Consequently:

$$
\hat{d}_{i j}=\hat{I}-\hat{\bar{\eta}}(M) \hat{\Gamma}^{j}(i)
$$

The convergence of these estimators based on the realization matrix has been studied and proved by Cao in [4].

The estimation of the matrix $D$ can also be computationally burdensome because it must be made for each couple $(i, j)$ (complexity of order $O\left(m^{2}\right)$ ). That is why an approximate estimate (with potential vector) is proposed in [5] which reduces the complexity of the calculation to the order $O(m)$.

Hence, thanks to the ergodicity of the process under consideration, the Perturbation Realization concept gives an estimate of the MDSM that:

- can be evaluated from a single sample path. This is very interesting from a prac- 
tical point of view for on-line performance optimization, when the parameters are impossible to change intentionally, or when the simulation of each perturbed path is computationally burdensome;

- can be evaluated without knowing the infinitesimal generator $M$ of the Markov process;

- can be evaluated in any direction by changing only the directional matrix Q.

\section{Application to reliability studies: numerical experiments}

The numerical results presented in this section are obtained with simulated operating feedback data. The aim is to show how the joint use of multi-directional sensitivity measure and the realization factors for their estimation can help in the sensitivity analysis of stationary performance in reliability studies. A first simple case is studied to make a connection between usual analytical results and estimation results with Perturbation Analysis in the case of Birnbaum's importance measure. Then, a more complex system is presented to enhance the advantages of multi directional sensitivity measure with Perturbation analysis. In both cases, the transition rate matrix is assumed to be unknown for the estimation, and the data set is made of the transition dates from one state to another. The performance measure is the asymptotic availability and the simulations are made for 100000 transitions.

\subsection{Binary-state systems with independent components}

\subsubsection{Expression of the performance function}

For binary-state systems, a performance function $\bar{\eta}(M)$ at steady state can be the availability, expressed as:

$$
\bar{\eta}(M)=\sum_{i \in \Omega_{o}} \pi_{i}
$$

with $\pi_{i}$ is the stationary probability of state $i$, and $\Omega_{o}$ is the set of the running states of the system. 


\subsubsection{Comparison of analytical and estimated results}

Consider first the two-unit system sketched in Figure 4. The availability of units $C_{1}, C_{2}$ at steady state are written as:

$$
a_{1}=\frac{\mu_{1}}{\lambda_{1}+\mu_{1}}, \quad a_{2}=\frac{\mu_{2}}{\lambda_{2}+\mu_{2}} .
$$

The asymptotic availability of the system can be obviously calculated with Kolmogorov equations at steady state:

$$
\begin{aligned}
-\left(\lambda_{1}+\lambda_{2}\right) \pi_{1}+\lambda_{1} \pi_{2}+\lambda_{2} \pi_{3} & =0 \\
\mu_{1} \pi_{1}-\left(\mu_{1}+\lambda_{2}\right) \pi_{2}+\lambda_{2} \pi_{4} & =0 \\
\mu_{2} \pi_{1}-\left(\lambda_{1}+\mu_{2}\right) \pi_{3}+\lambda_{1} \pi_{4} & =0 \\
\mu_{2} \pi_{2}+\mu_{1} \pi_{3}-\left(\mu_{1}+\mu_{2}\right) \pi_{4} & =0 \\
\pi_{1}+\pi_{2}+\pi_{3}+\pi_{4} & =1
\end{aligned}
$$

By solving these equations, the availability of the system is finally obtained:

$$
A=\pi_{1}+\pi_{2}+\pi_{3}=\frac{\mu_{1} \mu_{2}+\mu_{1} \lambda_{2}+\mu_{2} \lambda_{1}}{\left(\lambda_{1}+\mu_{1}\right)\left(\lambda_{2}+\mu_{2}\right)} .
$$

The derivative of $A$ with respect to $\lambda_{i}, \mu_{i}(i=1,2)$, can be obtained easily from this formula.

To compare with the estimated results, a set of data with transition instants $\lambda_{1}=$ $0.01, \lambda_{2}=0.01, \mu_{1}=0.05, \mu_{2}=0.05$ is generated. Then the realization matrix $D$ and the steady-state probability vector $\pi$ are estimated with Equation 10:

$$
\hat{D}=\left(\begin{array}{cccc}
0 & -1.3648 & -1.4528 & -11.2053 \\
1.3648 & 0 & -0.0850 & -9.8446 \\
1.4528 & 0.0850 & 0 & -9.8063 \\
11.2053 & 9.8446 & 9.8063 & 0
\end{array}\right)
$$

and

$$
\hat{\pi}=(0.6898,0.1406,0.1413,0.0283) .
$$

Consider now the perturbation on a parameter level, more precisely on $\lambda_{2}$, corresponding to the directional matrix $Q_{1}$ given in section 2.2.2. Then, using the estimators $\hat{D}$ and 
$\hat{\pi}$, Equation 8 gives:

$$
\begin{aligned}
& E\left(\hat{I}_{Q_{1}}\right)=E\left(\hat{I}_{Q\left(\lambda_{2}\right)}^{C}\right)=-2.3014, \\
& \operatorname{var}\left(\hat{I}_{Q_{1}}\right)=\operatorname{var}\left(\hat{I}_{Q\left(\lambda_{2}\right)}^{C}\right)=0.0357 .
\end{aligned}
$$

Figure 8 sketches the evolution of the estimator $\hat{I}_{Q\left(\lambda_{2}\right)}^{C}$ as a function of the sample size. Using Equation 5, the estimator $\hat{I}_{Q_{1}}$, and the analytical expression of $\frac{\partial a_{2}}{\partial \lambda_{2}}=-\frac{\mu_{2}}{\left(\lambda_{2}+\mu_{2}\right)^{2}}$, the estimated Birnbaum's importance measure equals:

$$
\hat{I}^{B}\left(C_{2}\right)=-E\left(\hat{I}_{Q\left(\lambda_{2}\right)}^{C}\right) \frac{\left(\lambda_{2}+\mu_{2}\right)^{2}}{\mu_{2}}=0.1657
$$

In comparison, using the analytical expression of the partial derivative $\partial A / \partial \lambda_{2}$, and Equations 4, one gets:

$$
\begin{aligned}
& \hat{I}_{Q\left(\lambda_{2}\right)}^{C}=\frac{\partial A}{\partial \lambda_{2}}=-2.3148, \\
& I^{B}\left(C_{2}\right)=0.1667 .
\end{aligned}
$$

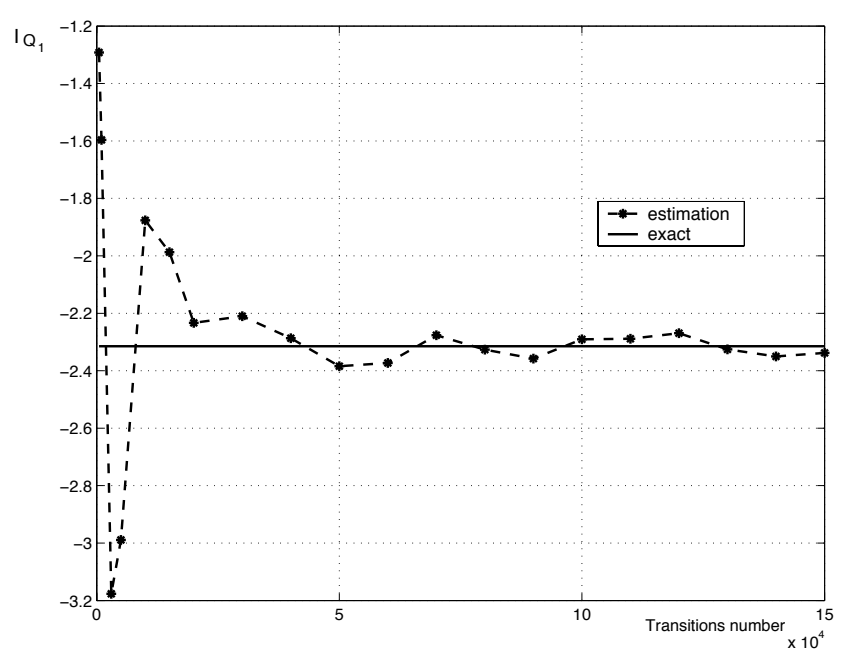

Figure 8: Estimator of $I_{Q_{1}}$ as a function of the sample size

For the case with the directional matrix $Q_{2}$ given in section 2.2.2, the perturbation corresponds to the perturbation on one specific state (state 3) and no more on one parameter. The sensitivity of the system availability in the direction $Q_{2}$ is obtained by using 
the same estimators $\hat{D}$, and $\hat{\pi}$ :

$$
\begin{aligned}
& E\left(\hat{I}_{Q_{2}}\right)=E\left(\hat{I}_{Q_{2}}^{S}\right)=3.2815 \\
& \operatorname{var}\left(\hat{I}_{Q_{2}}\right)=\operatorname{var}\left(\hat{I}_{Q_{2}}^{S}\right)=0.0288 .
\end{aligned}
$$

This derivative means that if the repair rate $\mu_{2}$ on state 3 is increased by an amount $3 \delta$, and at the same time, the failure rate $\lambda_{1}$ on state 3 is decreased by an amount $2 \delta$, then the system availability will increase by an amount $3.2815 \delta$. This value quantifies the gain for the system availability if the probability of being in state 3 is perturbed. Since state 3 is a running state and since the perturbation in the direction $Q_{2}$ increases the probability of in staying state 3 , the availability increases and $\hat{I}_{Q_{2}}^{S}$ indicates at which speed. To obtain the analytical result in this case, it would be necessary to rewrite the system of differential equations by distinguishing all transition rates between all states, for example to distinguish the parameter $\lambda_{1}$ representing the transition rate from state 1 towards state 2 , from the parameter $\lambda_{1}$ representing the transition rate from state 3 towards state 4 .

\subsection{Multi-state systems with dependent components}

\subsubsection{Presentation of the system}

This section concerns a more complex system. Figure 9 represents a part of a power system with 4 units divided into 2 groups:

- Generator group: units $C_{1}$ and $C_{2}$ are two generators, their capacities are $50 \mathrm{MW}$ (for normal operation state), 0 (for failed state). When $C_{2}$ is failed, the capacity of unit $C_{1}$ can increase by $20 \%$;

- Transformer group: units $C_{3}$ and $C_{4}$ are identical transformers, $C_{4}$ is in cold redundancy with $C_{3}$. As soon as $C_{3}$ is repaired, $C_{4}$ stops, and $C_{3}$ is the main operating unit. This means that when both units have failed, $C_{3}$ is repaired first. The nominal capacity values of $C_{3}$ and $C_{4}$ are $100 \mathrm{MW}$ and it can be changed synchronously with the power capacity from the generator group.

The operational mode of the system is described in Table 1 where "O" denotes operating state, "S" denotes standby state, and "F" denotes failed state. The corresponding Markov process and the distribution of the production capacity are drawn in Figure 10. 


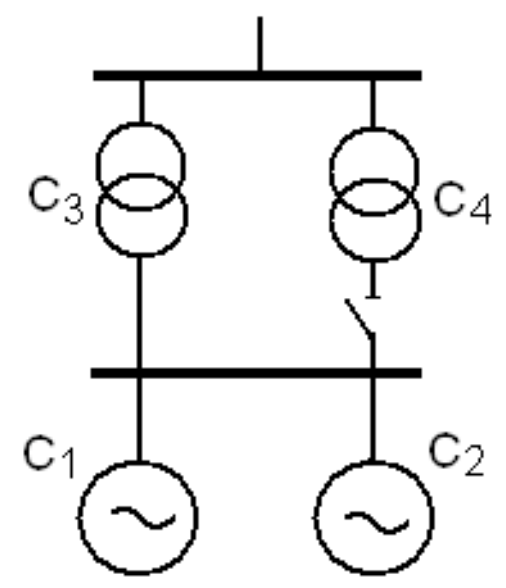

Figure 9: A part of power system

Table 2 gives the values of the repair rates $\mu_{i}$, the failure rates $\lambda_{i}, i=1, \ldots, 4$, as well as the production capacity. $\bar{\lambda}_{i}$ corresponds to failure rate in shared resource/load case.

\subsubsection{Performance function}

The multi-state systems (MSS), such as e.g. manufacturing, production lines, give different levels of performance that can settle on different values (e.g. $100 \%, 90 \%, 80 \%, \ldots$ of the nominal capacity) depending on the operative conditions of the constitutive multi-state components. The availability is considered to be a measurement of the ability of the system to meet the demand (required performance level), and the general definition of a multi-state system availability is [18]:

$$
A_{M S S}(t)=\operatorname{Pr}\left(W(t) \geq W^{*}\right),
$$

where $W(t)$ is the output performance of the MSS at time $t$, and $W^{*}$ is required MSS output performance. For renewable MSS, the stationary distribution of state probabilities is:

$$
\pi_{i}=\lim _{t \rightarrow \infty} \operatorname{Pr}\left(W(t)=W_{i}\right)
$$

where $W_{i}$ is the performance output of state $i$ with $i=1,2, \ldots, S$ ( $S$ represents the total number of possible system states). Then the steady state availability equals:

$$
\bar{\eta}(M)=A_{M S S}=\lim _{t \rightarrow \infty} A_{M S S}(t)=\sum_{i \in \Omega_{W^{*}}} \pi_{i},
$$




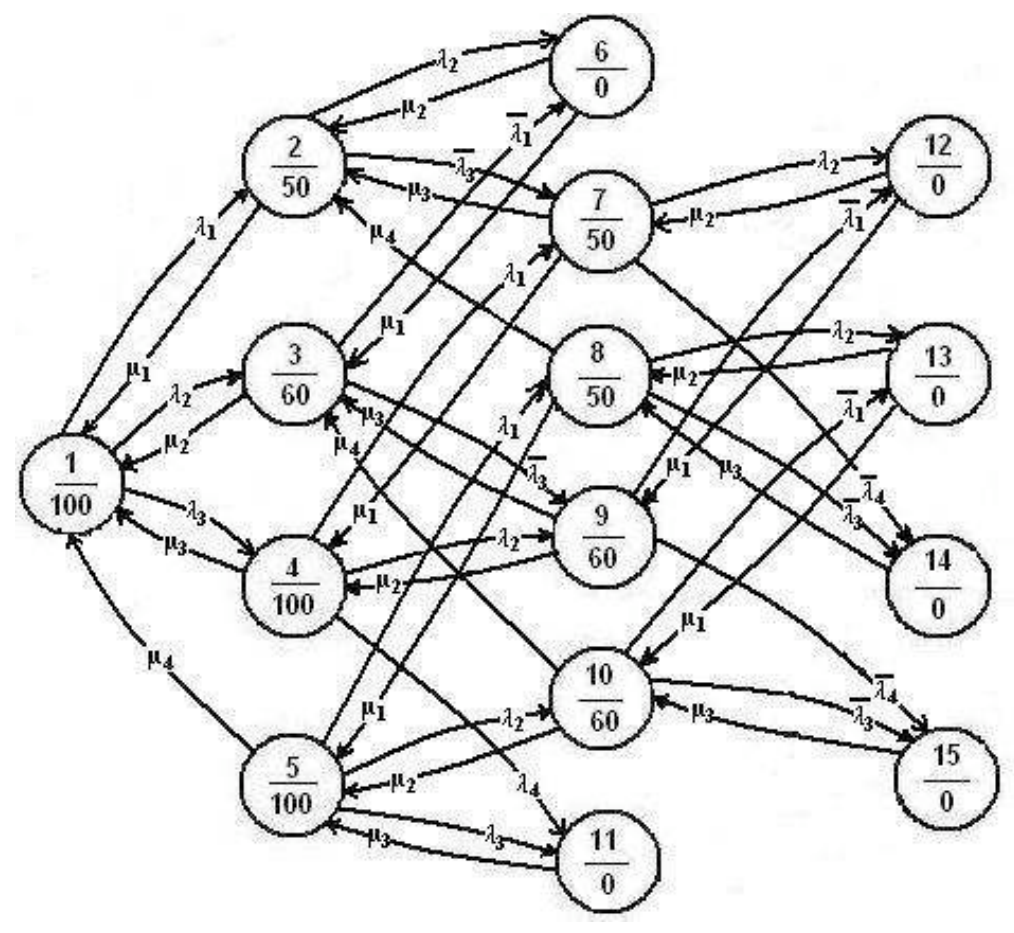

Figure 10: Markov process \& production capacities distribution

where $\Omega_{W^{*}}$ is a set of states $j$ whose performance output $W_{j} \geq W^{*}(j=1,2, \ldots, S)[26]$. $A_{M S S}$ is a function of the required performance level $W^{*}$ which is an additional indicator that has a strong impact on the importance of elements in multi-state systems. The availability of a certain component may be very important for one required performance level and less important for another [18].

\subsubsection{Numerical results}

In this case, the analytical calculation is long, thus, only the estimate results are presented. The data are simulated with parameter values given in Table 2 . The realization matrix $D$ and the steady state vector $\pi$ have been estimated once and all the results presented in Tables $3,4,5,6,7$, are obtained by changing only the directional matrix $Q$ in Equation 8.

\section{Sensitivity analysis to a parameter - $I_{Q}^{C}$}

First, the sensitivity of system availability with respect to the failure rates $\lambda_{i}, \bar{\lambda}_{i}$, and the repair rates $\mu_{i}(i=1, \ldots, 4)$ is studied. This corresponds to MDSM at the component level and to the specific directions $Q\left(\lambda_{i}\right), Q\left(\bar{\lambda}_{i}\right), Q\left(\mu_{i}\right)$. 
Table 1: System states

\begin{tabular}{|c|c|c|c|c|c|c|}
\hline \multirow[b]{2}{*}{ State } & \multicolumn{4}{|c|}{ Component } & \multirow[b]{2}{*}{ System } & \multirow[b]{2}{*}{ production capacity } \\
\hline & $C_{1}$ & $C_{2}$ & $C_{3}$ & $C_{4}$ & & \\
\hline 1 & $\mathrm{O}$ & $\mathrm{O}$ & $\mathrm{O}$ & $\mathrm{S}$ & $\mathrm{O}$ & 100 \\
\hline 2 & $\mathrm{~F}$ & $\mathrm{O}$ & $\mathrm{O}$ & $\mathrm{S}$ & $\mathrm{O}$ & 50 \\
\hline 3 & $\mathrm{O}$ & $\mathrm{F}$ & $\mathrm{O}$ & $\mathrm{S}$ & $\mathrm{O}$ & 60 \\
\hline 4 & $\mathrm{O}$ & $\mathrm{O}$ & $\mathrm{F}$ & $\mathrm{O}$ & $\mathrm{O}$ & 100 \\
\hline 5 & $\mathrm{O}$ & $\mathrm{O}$ & $\mathrm{O}$ & $\mathrm{F}$ & $\mathrm{O}$ & 100 \\
\hline 6 & $\mathrm{~F}$ & $\mathrm{~F}$ & $\mathrm{O}$ & $\mathrm{S}$ & $\mathrm{F}$ & 0 \\
\hline 7 & $\mathrm{~F}$ & $\mathrm{O}$ & $\mathrm{F}$ & $\mathrm{O}$ & $\mathrm{O}$ & 50 \\
\hline 8 & $\mathrm{~F}$ & $\mathrm{O}$ & $\mathrm{O}$ & $\mathrm{F}$ & $\mathrm{O}$ & 50 \\
\hline 9 & $\mathrm{O}$ & $\mathrm{F}$ & $\mathrm{F}$ & $\mathrm{O}$ & $\mathrm{O}$ & 60 \\
\hline 10 & $\mathrm{O}$ & $\mathrm{F}$ & $\mathrm{O}$ & $\mathrm{F}$ & $\mathrm{O}$ & 60 \\
\hline 11 & $\mathrm{O}$ & $\mathrm{O}$ & $\mathrm{F}$ & $\mathrm{F}$ & $\mathrm{F}$ & 0 \\
\hline 12 & $\mathrm{~F}$ & $\mathrm{~F}$ & $\mathrm{~F}$ & $\mathrm{O}$ & $\mathrm{F}$ & 0 \\
\hline 13 & $\mathrm{~F}$ & $\mathrm{~F}$ & $\mathrm{O}$ & $\mathrm{F}$ & $\mathrm{F}$ & 0 \\
\hline 14 & $\mathrm{~F}$ & $\mathrm{O}$ & $\mathrm{F}$ & $\mathrm{F}$ & $\mathrm{F}$ & 0 \\
\hline 15 & $\mathrm{O}$ & $\mathrm{F}$ & $\mathrm{F}$ & $\mathrm{F}$ & $\mathrm{F}$ & 0 \\
\hline
\end{tabular}

Table 2: Transition rates \& capacity.

\begin{tabular}{ccccc}
\hline Units & $\lambda_{i}$ & $\mu_{i}$ & $\bar{\lambda}_{i}$ & Capacity (MW) \\
\hline$C_{1}$ & $4.5 \mathrm{e}-4$ & $4 \mathrm{e}-3$ & $1 \mathrm{e}-3$ & $0 / 50 / 60$ \\
$C_{2}$ & $4.5 \mathrm{e}-4$ & $4 \mathrm{e}-3$ & - & $0 / 50$ \\
$C_{3}, C_{4}$ & $6.0 \mathrm{e}-4$ & $2 \mathrm{e}-3$ & $4.0 \mathrm{e}-4$ & $0 / 50 / 60 / 100$ \\
\hline
\end{tabular}

The results in Tables 3, and 4 show that an increase of failure rate leads to a decrease in system availability, and on the contrary, an increase in repair rate leads to an increase in availability. The sensitivity of system availability to the failure of $C_{j}(j=1,3,4)$ is shared between its sensitivity to $\bar{\lambda}_{j}$ and to $\lambda_{j}$, thus, the impact of the failure rate of $C_{j}$ on system sensitivity can be defined as: $I_{Q\left(\bar{\lambda}_{j}, \lambda_{j}\right)}^{C}=I_{Q\left(\bar{\lambda}_{j}\right)}^{C}+I_{Q\left(\lambda_{j}\right)}^{C}$.

Table 3 shows the results in the case with the required performance level $0<W^{*} \leq$ $50 \mathrm{MW}$. Considering the impact of component failure rate on system availability, $C_{3}$ is the most critical component, and the component importance ranking is: $C_{1}<C_{2}<C_{4}<C_{3}$. This ranking is also true considering the impact of component repair rate on the system availability. It can be partially explained by the system structure: $C_{3}$ is the main operating item of a generator group, therefore $C_{3}$ is more important than $C_{4} . C_{1}$ and $C_{2}$ are in a parallel structure, and $C_{4}$ is in a series structure (when $C_{4}$ is functioning, $C_{3}$ has already 
Table 3: Sensitivity analysis to failure \& repair rates, case $0<W^{*} \leq 50$

\begin{tabular}{c||ccc|ccc}
\hline Units & & Value & Order & & Value & Order \\
\hline \multirow{2}{*}{$C_{1}$} & $I_{Q\left(\lambda_{1}\right)}^{C}$ & -2.3270 & 4 & $I_{Q\left(\mu_{1}\right)}^{C}$ & 1.9277 & 4 \\
& $I_{Q\left(\bar{\lambda}_{1}\right)}^{C}$ & -8.4298 & 4 & & & \\
\hline \multirow{2}{*}{$C_{2}$} & $I_{Q\left(\lambda_{2}\right)}^{C}$ & -23.4837 & 3 & $I_{Q\left(\mu_{2}\right)}^{C}$ & 3.1516 & 3 \\
\hline \multirow{2}{*}{$C_{3}$} & $I_{Q\left(\lambda_{3}\right)}^{C}$ & -78.0152 & 1 & $I_{Q\left(\mu_{3}\right)}^{C}$ & 42.0794 & 1 \\
& $I_{Q\left(\bar{\lambda}_{3}\right)}^{C}$ & -16.0406 & & & \\
\hline \multirow{2}{*}{$C_{4}$} & $I_{Q\left(\lambda_{4}\right)}^{C}$ & -61.6264 & 2 & $I_{Q\left(\mu_{4}\right)}^{C}$ & 4.5391 & 2 \\
& $I_{Q\left(\bar{\lambda}_{4}\right)}^{C}$ & -12.3478 & 2 & & \\
\hline
\end{tabular}

Table 4: Sensitivity analysis to failure \& repair rates, case $50<W^{*} \leq 60$

\begin{tabular}{c||ccc|ccc}
\hline Units & & Value & Order & & Value & Order \\
\hline \multirow{2}{*}{$C_{1}$} & $I_{Q\left(\lambda_{1}\right)}^{C}$ & -163.8876 & 1 & $I_{Q\left(\mu_{1}\right)}^{C}$ & 22.6118 & 1 \\
& $I_{Q\left(\bar{\lambda}_{1}\right)}^{C}$ & -16.1538 & & & \\
\hline \multirow{2}{*}{$C_{2}$} & $I_{Q\left(\lambda_{2}\right)}^{C}$ & -13.8236 & 4 & $I_{Q\left(\mu_{2}\right)}^{C}$ & 1.2707 & 4 \\
\hline \multirow{2}{*}{$C_{3}$} & $I_{Q\left(\lambda_{3}\right)}^{C}$ & -71.0226 & 2 & $I_{Q\left(\mu_{3}\right)}^{C}$ & 20.7130 & 2 \\
& $I_{Q\left(\bar{\lambda}_{3}\right)}^{C}$ & -13.8683 & & & & \\
\hline \multirow{2}{*}{$C_{4}$} & $I_{Q\left(\lambda_{4}\right)}^{C}$ & -55.7066 & 3 & $I_{Q\left(\mu_{4}\right)}^{C}$ & 4.1319 & 3 \\
& $I_{Q\left(\bar{\lambda}_{4}\right)}^{C}$ & -10.9899 & 3 & & \\
\hline
\end{tabular}

failed) so the impact of $C_{4}$ on system availability behavior is more important than $C_{1}$ and $C_{2}$.

The results of sensitivity analysis in the case of the required performance level $50<$ $W^{*} \leq 60 \mathrm{MW}$ are presented in Table 4 . In this case, the set of functioning states of system is $\Omega_{W^{*}}=\{1,3,4,5,9,10\}$ (see Figure 10). According to the results of importance measures showed in Table 4, the new components importance ranking related to failure rates is: $C_{2}<C_{4}<C_{3}<C_{1}$. The maximum capacity of $C_{2}$ is only $50 \mathrm{MW}$, hence if $C_{1}$ fails the system is unable to supply the demand $W^{*}$ and it is considered as failed. Consequently, component $C_{1}$ ranks first and $C_{2}$ becomes the least important one. $C_{3}$ is still more important than $C_{4}$ since $C_{3}$ is the main operating unit of the standby structure.

The results in Tables 3 and 4 show that the component importance ranking of multi- 
Table 5: Sensitivity analysis to a group of parameters

\begin{tabular}{ccc|cc}
\hline & \multicolumn{2}{c|}{ Case $0<W^{*} \leq 50$} & \multicolumn{2}{c}{ Case $50<W^{*} \leq 60$} \\
\cline { 2 - 5 } Directions & $I_{Q\left(\lambda_{i}, \lambda_{j}\right)}^{C}$ & Order & $I_{Q\left(\lambda_{i}, \lambda_{j}\right)}^{C}$ & Order \\
\hline$Q\left(\lambda_{1}, \lambda_{2}\right)$ & -34.2405 & 6 & -193.8651 & 3 \\
$Q\left(\lambda_{1}, \lambda_{3}\right)$ & -104.8126 & 3 & -264.9323 & 1 \\
$Q\left(\lambda_{1}, \lambda_{4}\right)$ & -84.7310 & 5 & -246.7380 & 2 \\
$Q\left(\lambda_{2}, \lambda_{3}\right)$ & -113.5395 & 2 & -98.7145 & 5 \\
$Q\left(\lambda_{2}, \lambda_{4}\right)$ & -97.4579 & 4 & -80.5201 & 6 \\
$Q\left(\lambda_{3}, \lambda_{4}\right)$ & -168.0301 & 1 & -151.5874 & 4 \\
\hline
\end{tabular}

state systems depends not only on the system structure, on the component behaviour but also on the required performance level $W^{*}$.

\section{Sensitivity analysis to a group of parameters - $I_{Q}^{C}$}

Here, the perturbations on a group of parameters are studied. The sensitivity level in these directions can be used to identify the importance of components in a group. In Table 5 , directions $Q\left(\lambda_{i}, \lambda_{j}\right)$ with $i, j=1, . ., 4$ are denoted to indicate that the failure rate of both different components $C_{i}, C_{j}$ are perturbed by the same small amount $\delta$ (i.e. $\left.Q\left(\lambda_{i}, \lambda_{j}\right)=Q\left(\lambda_{i}\right)+Q\left(\lambda_{j}\right)\right)$. The multi-directional sensitivity measure $I_{Q\left(\lambda_{i}, \lambda_{j}\right)}^{C}$ gives an indicator of the system availability sensitivity to a group of components $\left(C_{i}, C_{j}\right)$.

- Application for groups importance ranking:

According to these results in Table 5, the groups/components importance ranking can be identified. When the required performance level is $0<W^{*} \leq 50$, the most important group of 2 components is $\left(C_{3}, C_{4}\right)$ and the group $\left(C_{1}, C_{2}\right)$ is the least important one. When the required performance level is $50<W^{*} \leq 60$, the most important group is $\left(C_{1}, C_{3}\right)$ and the $\left(C_{2}, C_{4}\right)$ is the least important group. These rankings can be also verified by adding the results showing in Tables 3,4 .

- Application to maintenance optimization: When one parameter of the system is changed (increased failure rate, component degradation, for example), the system availability deteriorates. This variation can be compensated completely or partially if at the same time, other parameters of the system (repair rates, for example) can be perturbed to compensate for this change. This action can be performed by choosing a suitable direction of perturbation $Q$. More precisely, now assume that the failure rate of $C_{2}$ is increased by an amount $\delta$ (degradation of component 2), 
Table 6: Sensitivity analysis to the failure rate of a given state

\begin{tabular}{ccc|cc}
\hline & \multicolumn{2}{c|}{ Case $0<W^{*} \leq 50$} & \multicolumn{2}{c}{ Case $50<W^{*} \leq 60$} \\
\cline { 2 - 5 } Directions & $I_{Q_{S i}}^{S}$ & Order & $I_{Q_{S i}}^{S}$ & Order \\
\hline$Q_{S 1}$ & -72.0632 & 1 & -193.6379 & 1 \\
$Q_{S 2}$ & -15.5054 & 4 & - & - \\
$Q_{S 3}$ & -12.8284 & 5 & -17.9662 & 4 \\
$Q_{S 4}$ & -62.8318 & 2 & -83.3452 & 2 \\
$Q_{S 5}$ & -16.5918 & 3 & -24.4980 & 3 \\
$Q_{S 7}$ & -8.4966 & 6 & - & - \\
$Q_{S 8}$ & -2.3335 & 8 & - & - \\
$Q_{S 9}$ & -6.9902 & 7 & -7.7539 & 5 \\
$Q_{S 10}$ & -1.6298 & 9 & -2.2025 & 6 \\
\hline
\end{tabular}

and at the same time, the repair rate of $C_{2}, \mu_{2}$, is perturbed by an amount $\alpha \delta$ (reduction of the maintenance time of $C_{2}$ ) . A sensitivity close to zero in a direction of the form $\left(\lambda_{2}, \alpha \mu_{2}\right)$ indicates that the change on $\mu_{2}$ almost balances the effect of the change on $\lambda_{2}$. Herein, for the case $0<W^{*} \leq 50: I_{Q\left(\lambda_{2}, 7.45 \mu_{2}\right)}^{C}=-0.0042$, and when $50<W^{*} \leq 60: I_{Q\left(\lambda_{2}, 10.88 \mu_{2}\right)}^{C}=0.0016$. From a practical point of view, this can be seen as a means to tune the maintenance parameters, such in a way that a perturbation on the failure rate has no impact on system availability. Maintenance policy parameters can then be optimally tuned in this way and the optimal solution can also depend on other criteria (maintenance cost, for example).

\section{Sensitivity analysis to failure rates in a system state - $I_{Q}^{S}$}

In this paragraph, composite directions of sensitivity are considered, i.e. the perturbation is not limited to a single specific parameter or group parameters. Instead, perturbations on the failure rates of a given state are considered. In Table 6 , the direction denoted $Q_{S i}$ indicates that all failure rate transitions out from the operational state $i$ $(i=1,2,3,4,5,7,8,9,10)$ are simultaneously perturbed by the same amount. The sensitivity in these directions can help to identify the importance of a given state. According to these sensitivities with the different required performance levels, the different state importance rankings are drawn. It is shown that, for both cases: $0<W^{*} \leq 50$ and $50<W^{*} \leq 60$, the most important state is state number 1 and state 10 is the least important one. 
Table 7: Sensitivity analysis to the failure rates of a group of states

\begin{tabular}{cccc|ccc}
\hline & \multicolumn{3}{c|}{ Case $0<W^{*} \leq 50$} & \multicolumn{3}{c}{ Case $50<W^{*} \leq 60$} \\
\cline { 2 - 7 } Directions & States & $I_{Q_{\left(C_{i} C_{j}\right)}}^{S}$ & Order & States & $I_{Q_{\left(C_{i} C_{j}\right)}}^{S}$ & Order \\
\hline$Q_{\left(C_{1} C_{2}\right)}$ & $1,4,5$ & -15.0073 & 5 & $1,4,5$ & -175.6364 & 2 \\
$Q_{\left(C_{1} C_{3}\right)}$ & $1,3,5,10$ & -96.1990 & 2 & $1,3,5,10$ & -228.4462 & 1 \\
$Q_{\left(C_{1} C_{4}\right)}$ & 4,9 & -66.7465 & 4 & 4,9 & -90.9881 & 3 \\
$Q_{\left(C_{2} C_{3}\right)}$ & $1,2,5,8$ & -107.6237 & 1 & 1,5 & -82.6828 & 4 \\
$Q_{\left(C_{2} C_{4}\right)}$ & 4,7 & -71.0516 & 3 & 4 & -55.7766 & 5 \\
\hline
\end{tabular}

\section{Sensitivity analysis to failure rates in a group of states - $I_{Q}^{S}$}

This paragraph explores the sensitivity of the failure rates of a group of sates. In Table 7 , the direction denoted $Q_{\left(C_{i} C_{j}\right)}(i, j=1,2,3,4)$ indicates that when the system is in operational state where both $C_{i}, C_{j}$ are functioning, the failure rates of $C_{i}, C_{j}$ are simultaneously perturbed by the same amount. From a practical point of view, this perturbation could be caused by, for example, electrical shock, changing environmental conditions, etc. The sensitivity of system availability in this direction gives impact of the operational components group on system availability.

The results in Table 7 show that with the different required performance levels, the sensitivity of system availability to operational component groups is different. When the required performance level is $0<W^{*} \leq 50$, the most important group of 2 operational components is $\left(C_{2}, C_{3}\right)$ and the group $\left(C_{1}, C_{2}\right)$ is the least important one. And if the required performance level is $50<W^{*} \leq 60$, the most important group is $\left(C_{1}, C_{3}\right)$ and the group $\left(C_{2}, C_{4}\right)$ is the least important one.

\section{Conclusions}

The results presented in this paper are a natural extension of the classical methods of sensitivity analysis developed for "static systems". The main idea is to obtain the derivatives of a performance measure without using exact or approximate methods which are burdensome, neither FD/SP methods which require data from both the nominal and the perturbed system behaviour. In fact, the data of the perturbed system can be unavailable in many real-life cases when the parameters cannot be intentionally modified (for economic or safety reasons for example). 
With PA and IPA, methods have been developed to estimate the sensitivity measure of discrete event dynamic system models on the basis of nominal system behavior only. In the framework of Markov process modeling, the Perturbation Realization approach based on a single sample path is particularly well formalized. From a practical point of view, it allows the estimation of sensitivity measures on the basis of operating feedback data in nominal conditions, without knowing the generator of the underlying Markov process. The present work shows that after one Perturbation Realization matrix estimation, many different sensitivity measures, sensitivity to one or more parameters with any directional derivative, sensitivity to the transition rates, can be led with no additional calculations and can be used in many reliability studies: identification of a group of critical components, quantification of the impact of a component failure in a given state, adaptation of the maintenance parameters to keep a constant availability level in case of components degradation,etc...

This paper is the development of our research in the framework of the sensitivity importance analysis of dynamic systems presented in part in [10]. Our further research will focus on more detailed applications of Perturbation Realization to the sensitivity studies of dynamic systems and the development of methods to analyze the transient state of a Markov processes.

\section{References}

[1] E. Borgonovo and G.E. Apostolakis. A new importance measure for risk-informed decision making. Reliability Engineering and System Safety, 72(2):193-212, 2001.

[2] E. Borgonovo, G.E. Apostolakis, S. Tarantola, and A. Saltelli. Comparison of global sensitivity analysis techniques and importance measures in PSA. Reliability Engineering and System Safety, (79):175-185, 2003.

[3] X.-R Cao. Perturbation analysis of discrete event systems: Concepts, algorithms and applications. European Journal of Operation Research, 91:1-13, 1995.

[4] X.-R. Cao and H.-F. Chen. Pertubation realization, potentials, and sensitivity analysis of markov processes. IEEE Transactions on Automatic Control, 42(10):1382-1393, 1997. 
[5] X.-R. Cao and Y.-W. Wan. Algorithms for sensitivity analysis of markov systems through potential and perturbation realization. IEEE Transactions on Automatic Control, 6(4):482-494, 1998.

[6] X.-R Cao, X.M Yuan, and L. Qiu. A single sample path-based performance sensitivity formula for Markov chains. IEEE Transactions on Automatic Control, 41(12):1814$1817,1996$.

[7] L. Dai. Sensitivity analysis of stationary performance measures for Markov chains. Mathematical and Computer Modelling, 23(11-12):143-160, 1996.

[8] L. Dai. Rate of convergence for derivative estimation of discrete-time Markov chains via finite-difference approximation with common random numbers. SIAM Journal on Applied Mathematics, 57(3):731-751, 1997.

[9] L. Dai and C.-Y. Ho. Structural infinitesimal perturbation analysis (SIPA) for derivative estimation of discrete-event dynamic systems. IEEE Transactions on Automatic Control, 40(7):1154-1166, 1995.

[10] P. Do Van, S. Khalouli, A. Barros, and C. Berenguer. Sensitivity \& importance analysis of markov models using perturbation analysis: Applications in reliability. In C. Guedes-Soares and E. Zio, editors, Safety and Reliability for Managing Risk Proc. of ESREL 2006, 18-22 sep. 2006, Estoril, Portugal, pages 1769-1775. Taylor \& Francis, 2006.

[11] Y. Dutuit and A. Rauzy. Efficient algorithms to assess component and gate importance in fault tree analysis. Reliability Engineering and System Safety, 72:213-222, 2001.

[12] P. Frank. Introduction to system sensitivity. Academic press, 1978.

[13] M.C. Fu and J.Q. Hu. Smoothed perturbation analysis derivative estimation for Markov chains. Operations Research Letters, 15:241-251, 1994.

[14] P. Glasserman. Derivative estimates from simulation of continuous-time Markov chains. Operations Research, 40(2):292-308, 1992.

[15] S. Glasserman, P. Tayur. Sensitivity analysis for base-stock levels in multiechelon production-inventory systems. Management Science, 41(2), 1995.

[16] P.W. Glynn. Likelihood ratio gradient estimation for stochastic systems. Communications of the ACM, 33:75-84, 1990. 
[17] A.B. Huseby. Importance measures for multicomponent binary systems. Technical Report Statistical Research Report No. 11-04 - ISSN 0806-3842, University of Oslo Dept. of Math., December 2004.

[18] G. Levitin and A. Lisnianski. Importance and sensitivity analysis of multi-state systems using the universal generating function method. Reliability Engineering and System Safety, 65:271-281, 1999.

[19] B. Natvig. Reliability: Importance of components. Encyclopedia of Statistical Sciences, (8), 1988.

[20] Y. Ou and J. Bechta-Dugan. Approximate sensitivity analysis for acyclic Markov reliability models. IEEE Transactions on Reliability, 52(2):220-231, 2003.

[21] G.C. Pflug. Sampling derivatives of probabilities. Computing, 42:315-328, 1989.

[22] G.C. Pflug. On-line optimization of simulated markovian processes. Mathematics of Operations Research, 15:381-395, 1990.

[23] M. Rausand and H. Hoyland. System Reliability Theory - Models, Statistical methods and Application. Wiley Series in Probability and Statistics. Wiley Interscience, second edition edition, 2004.

[24] S. Ross. Stochastic Processes. Wiley Series in Probability and Statistics. John Wiley \& Sons, Inc., 1996.

[25] J.C. Spall. Multivariate stochastic approximation using a simultaneous perturbation gradient approximation. IEEE Transactions on Automatic Control, 37(4):332-341, 1992.

[26] E. Zio and L. Podofillini. Importance measures of multi-state components in multistate systems. International Journal of Reliability, Quality and Safety Engineering, 10(3):289-310, 2003. 